\title{
IV Abkürzungsverzeichnis
}

ABiSA Allgemeiner Behindertenverband in Sachsen-Anhalt

AEP Ansatz eigendynamischer politischer Prozesse

AKH Architekten- und Stadtplanerkammer

ARGEBAU Arbeitsgemeinschaft der für das Bau-, Wohnungs- und Siedlungswesen zuständigen Minister und Senatoren der Länder (»Bauministerkonferenz«)

AWI Arbeitsgemeinschaft der Wohnungs- und Immobilienverbände

AZI Akteurzentrierter Institutionalismus

BauO LSA Bauordnung des Landes Sachsen-Anhalt

BDB Bund deutscher Baumeister, Architekten und Ingenieure

BFW Bundesverband Freier Immobilien- und Wohnungsunternehmen

BGG Behindertengleichstellungsgesetz

BMI Bundesinnenministerium

BSBH Blinden- und Sehbehindertenbund

BAG Bundesarbeitsgemeinschaft

CBF Club Behinderter und ihrer Freunde

DIN Deutsches Institut für Normung

HBO Hessische Bauordnung

HessBGG Hessisches Behindertengleichstellungsgesetz

HFW Hessische Fachstelle für Wohnberatung

HMSI Hessisches Ministerium für Soziales und Integration

HMWEVW Hessisches Ministerium für Wirtschaft, Energie, Verkehr und Wohnen

HWB Hofheimer Wohnungsbau GmbH

IHK Industrie- und Handelskammer

IP Interviewpartner* in

KfW Kreditanstalt für Wiederaufbau

KIP Kommunalinvestitionsprogramm

LAG Landesarbeitsgemeinschaft

LBO Landesbauordnung

LFI Landesförderinstitut

MBO Musterbauordnung

Merkzeichen aG Merkzeichen »außergewöhnlich gehbehindert» 
Merkzeichen BI Merkzeichen »blind«

Merkzeichen H Merkzeichen »hilflos«

MLV Ministerium für Landesentwicklung und Verkehr, Sachsen-Anhalt

MSA Ministerium für Arbeit, Soziales und Integration, Sachsen-Anhalt

MVV TB Muster Verwaltungsvorschrift Technische Baubestimmungen

PiA Prävention im Alter

UN-BRK UN-Behindertenrechtskonvention

VdK Ursprünglich: Verband der Kriegsbeschädigten, Kriegshinterbliebenen und Sozialrentner Deutschlands e. V.

VdW Verband der Wohnungswirtschaft

VdWg Verband der Wohnungsgenossenschaften

VhU Vereinigung der hessischen Unternehmerverbände

VV TB Verwaltungsvorschrift Technische Baubestimmungen

WEG Wohnungseigentümergemeinschaften

WIP Wohnrauminvestitionsprogramm 